\section{Flies put the buzz back into long-term-potentiation}

Ole Paulsen and Richard G. M. Morris

\author{
Two new papers show that an atypical protein kinase $\mathbf{C}$ may \\ mediate the maintenance of long-term potentiation in the \\ mouse hippocampus and of associative memory in Drosophila.
}

What maintains our memories? How can we recall events from long ago, or facts learned early in our lives, despite massive turnover of the molecules of memory? Two lines of evidence presented in this issue implicate an atypical form of protein kinase $\mathrm{C}$ (aPKC) in the maintenance of memory. First, Drier et al. ${ }^{1}$ show that induction of the corresponding mouse transgene enhances memory in an associative memory task in Drosophila. Second, Ling et al. ${ }^{2}$ demonstrate that atypical PKC is necessary for maintaining hippocampal long-term potentiation (LTP). Despite potentially important differences in the time scales over which these two studies were done (24 and 6 hours, respectively), these findings point to an evolutionarily conserved signal-transduction cascade that contributes to the persistence of memory.

Protein kinases are typically activated by second messengers such as cAMP, calcium and phospholipids, and some kinases occur in autonomous forms. For example, calcium/calmodulin-dependent protein kinase II (CaMKII) can undergo autophosphorylation and become calcium independent. This intriguing property of CaMKII has been proposed as a 'molecular switch' that underlies memory ${ }^{3}$. The calciumand phospholipid-dependent protein kinase (PKC) also exists in a persistently active form. PKC contains a regulatory domain with a pseudosubstrate sequence covering the catalytic site (Fig. 1a). Upon activation, the catalytic domain is exposed via a conformational change induced by the binding of second messengers. Proteolytic cleavage

\footnotetext{
Ole Paulsen is in the Department of Physiology, Oxford University, Parks Road, Oxford OX1 3PT, UK. Richard Morris is in the Department of Neuroscience, University of Edinburgh, 1 George Square, Edinburgh, EH8 9JZ, UK.

e-mail:r.g.m.morris@ed.ac.uk
}

leaves the catalytic domain constitutively active because it is not inhibited by the pseudosubstrate sequence. This persistently active form of PKC is called PKM. Calcium-dependent proteolysis leading to persistent activation of PKC has been proposed as a mechanism for the maintenance of $\mathrm{LTP}^{4}$.

Genetic screening has identified at least twelve distinct members of the PKC family of enzymes that can be sorted into three main categories: conventional PKC, novel PKC and atypical $\mathrm{PKC}^{5}$. The subject of these new studies
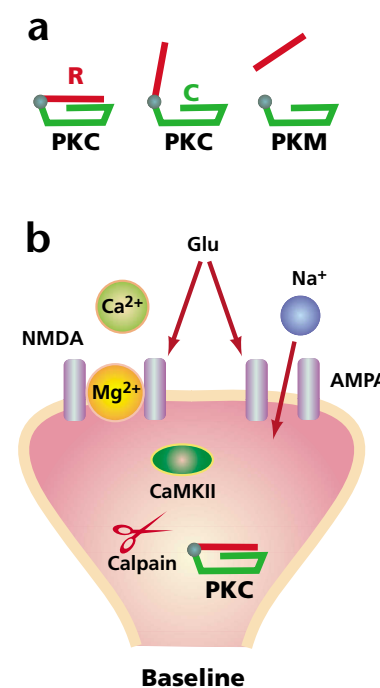
AMPA receptor channels. is one of the aPKCs, the so-called $\mathrm{PKC} \zeta$. It too comes in a persistently active or 'truncated' form, generally called aPKM. Armed with new molecular biological tools as well as increased understanding of the PKC enzyme family, the authors of these two studies have re-visited the role of PKC in memory and plasticity.

Drier et al. ${ }^{1}$ studied memory after odor-avoidance conditioning in flies. One odor is presented together with an electric shock. A different odor is presented alone. Following training, the flies are simultaneously exposed to both odors. Learning is indicated by the avoidance of the shock-paired odor. Memory is measured as the persistence of this behavior over 24 hours. To study the effect of the persistently active form of PKC- $\zeta(\mathrm{aPKM})$ on this behavior, an aPKM transgene was introduced into Drosophila under the control of a heatshock promoter ${ }^{1}$. The authors found that induction of this transgene or the Drosophila homologue after training
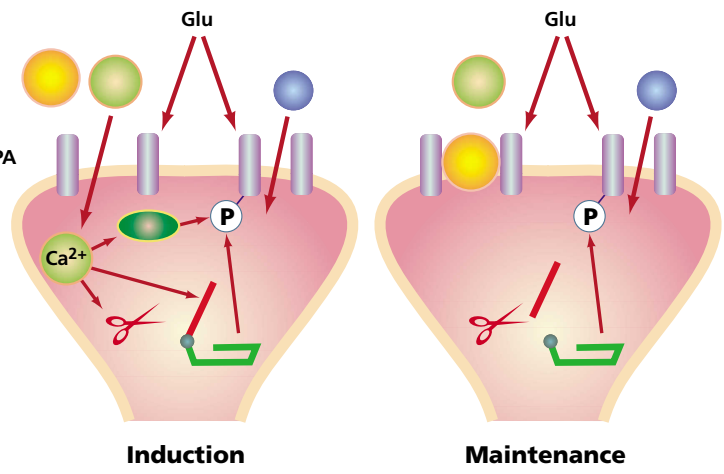

Don $r \cos ^{\mathrm{n} n}$

Fig. I. Role of PKM in LTP. (a) Schematic drawing of protein kinase $C$ in its resting configuration (left), activated state (middle) and persistently active form (right). In its resting configuration, a pseudosubstrate sequence in the regulatory domain $(R)$ inhibits the catalytic domain. The catalytic domain (C) is exposed via a conformational change upon activation by different second messengers. Proteolytic cleavage leaves the catalytic domain (PKM) constitutively active because it is not inhibited by the pseudosubstrate sequence. (b) Model for induction and maintenance of LTP. During baseline conditions, glutamate (Glu) released from the presynaptic bouton binds to both AMPA and NMDA receptors on the postsynaptic spine. However, the EPSP is mediated exclusively via AMPA receptors, as NMDA receptors are blocked by $\mathrm{Mg}^{2+}$ ions. During induction of LTP, depolarization of the postsynaptic cell (by converging excitatory inputs or backpropagating action potentials) relieves the $\mathrm{Mg}^{2+}$ block of the NMDA receptor channel, allowing $\mathrm{Ca}^{2+}$ ions into the postsynaptic spine. The resultant $\mathrm{Ca}^{2+}$ increase triggers LTP by activating protein kinases such as CaMKII and PKC. These are necessary during the induction of LTP. In parallel, atypical PKC is turned into a persistently active form via $\mathrm{Ca}^{2+}$ activated proteolytic cleavage (calpain). During maintenance, the AMPA receptors remain phosphorylated by persistent kinase activity (PKM), leading to increased conductance of 
enhanced memory. Moreover, either an apparently specific inhibitor of aPKM called chelerythrine or the induction of a dominant-negative mouse aPKM transgene caused faster forgetting without affecting the initial ability to learn. These results are important, as they suggest how the often-seen dissociation between initial learning and later memory might be understood at a molecular level.

Whereas flies are suitable for studying genes involved in memory, they are less suitable for addressing its cellular mechanisms. To gain insight into these mechanisms, Ling et al. ${ }^{2}$ turned to hippocampal LTP. LTP is arguably the beststudied example of synaptic plasticity in the mammalian brain and an attractive model for associative learning ${ }^{6,7}$. Intense study of the underlying molecular mechanisms has shown that it encompasses several processes and temporal stages (induction, expression and maintenance), which are mediated by a multitude of different biochemical mechanisms ${ }^{6}$. There is general agreement that the induction of associative LTP is triggered by calcium influx through NMDA receptors ${ }^{6}$ (Fig. 1b). The expression mechanisms are hotly debated, in particular whether the locus of change is presynaptic, postsynaptic or some combination of both. Phosphorylation of AMPA receptors is one possible candidate for a postsynaptic mechanism $^{8}$. Maintenance mechanisms have so far been much less studied, but a persistently active protein kinase, such as autophosphorylated $\mathrm{CaMKII}^{3}$ or $\mathrm{PKM}^{4}$, would fit the bill.

Ling et al. ${ }^{2}$ conclude that aPKM is both necessary and sufficient for the persistence of potentiation over six hours ${ }^{2}$. Exogenous introduction of recombinant aPKM into the postsynaptic neuron produced a persistent potentiation that then occluded the subsequent induction of activity-induced LTP. Both this potentiation and regular LTP could be reversed by the selective inhibitor chelerythrine. Importantly, the aPKM-induced potentiation was observed during blockade of NMDA receptors, thereby excluding an indirect effect via an enhancement of an NMDA receptor-mediated current. Further experiments, using different kinase inhibitors, investigated whether aPKM was necessary for the persistence of LTP. Whereas staurosporine, a general kinase inhibitor, blocked the induction of LTP at concentrations below those blocking aPKM, it had no effect on recently established LTP. In contrast, both chelerythrine and a pseudosubstrate inhibitor, at concentrations that selectively blocked atypical PKCs (including aPKM), completely reversed pre-established LTP without any effect on a nonpotentiated control input. These results also suggest that the molecular machinery responsible for the initial maintenance of LTP is already present at the site of induction, rather than being transported from some other location in the cell, and that this process is rapidly activated, for example via proteolytic cleavage of aPKC. Interestingly, inhibition of the calcium-activated protease calpain was reported to block the induction of LTP9 9 . This does not preclude the possibility, of course, that later stages of LTP maintenance could be mediated via freshly arriving protein synthesized elsewhere in the cell ${ }^{10}$.

However, aPKC may also be involved in induction. The introduction of chelerythrine and a dominant negative inhibitory form of aPKM into the postsynaptic neuron also completely eliminated induction ${ }^{2}$. Thus, aPKC might have a wider role than merely LTP persistence-it might also be an obligatory mediator in the signaling cascade leading to LTP induction (at least in the experimental preparation used, hippocampal slices from Sprague-Dawley rats at postnatal days 18-30).

These results might stir up some controversy, as much evidence up to now has favored CaMKII as a mediator of LTP rather than a PKC isoform ${ }^{8,11}$. Application of CaMKII inhibitors block the induction of $\mathrm{LTP}^{12,13}$, and a constitutively active form of CaMKII applied intracellularly results in a potentiation that occludes subsequent attempts at inducing $\mathrm{LTP}^{14}$. Ling et al. ${ }^{2}$ argue that this potentiating effect of intracellular CaMKII might be confounded by the need to add protein phosphatase inhibitors during such experiments ${ }^{2}$. However, involvement of PKM in maintaining LTP does not preclude a role for CaMKII during the initial induction or early expression of LTP. Indeed, PKC and CaMKII share a phosphorylation site on the GluR1 subunit of AMPA receptors ${ }^{15}$. There is, however, a fundamental difference between the autophosphorylation and the proteolysis mechanisms for producing persistent kinase activity. Whereas the former can be reversed by phosphatase activity, the latter is irreversible. Varying induction conditions could recruit one or other of these mechanisms, with recruitment of the proteolysis mechanism likely to reduce substantially the amount of depotentiation or long-term depression that can later be induced at the same synapses (thereby helping us to understand another controversy in the literature).

Although we should be cautious not to overinterpret these new data, they raise interesting new avenues for studying the complex relationships among signal transduction pathways, the expression and maintenance of synaptic plasticity, and thus the persistence of memory over time. By elucidating the details of the cellular and molecular machinery involved in LTP, we step further along the yellow brick road toward a deeper understanding of one of the fundamental problems of modern neuroscience ${ }^{7}$.

1. Drier, E. A. et al. Nature Neurosci. 5, 316-324 (2002).

2. Ling, D. S. F. et al. Nature Neurosci. 5, 295-296 (2002).

3. Lisman, J. E. Proc. Natl. Acad. Sci. USA 82, 3055-3057 (1985).

4. Malinow, R., Madison, D. V. \& Tsien, R. W. Nature 335, 820-824 (1988).

5. Mellor, H. \& Parker, P. J. Biochem J. 332, 281-292 (1998).

6. Bliss, T. V. P. \& Collingridge, G. L. Nature 361, 31-39 (1993).

7. Martin, S. J., Grimwood, P. D. \& Morris, R. G. M. Annu. Rev. Neurosci. 23, 649-711 (2001).

8. Malenka, R. C. \& Nicoll, R. A. Science 285, 1870-1874 (1999).

9. Staubli, U., Larson, J., Thibault, O., Baudry, M. \& Lynch, G. Brain Res. 444, 153-158 (1988).

10. Frey, U. \& Morris, R. G. M. Trends Neurosci. 21, 181-188 (1998).

11. Lisman, J., Schulman, H, \& Cline, H. Nat. Rev. Neurosci. 3, 175-190 (2002).

12. Malenka, R. C. et al. Nature 340, 554-557 (1989).

13. Malinow, R., Schulman, H. \& Tsien, R. W. Science 245, 862-866 (1989).

14. Lledo, P. M. et al. Proc. Natl. Acad. Sci. USA 92, 11175-11179 (1995).

15. Barria, A., Derkach, V. \& Soderling, T. J. Biol. Chem. 272, 32727-32730 (1997). 